\title{
Optimization Model for Integrated Municipal Solid Waste System Using Stochastic Chance-Constraint Programming under Uncertainty: A Case Study in Qazvin, Iran
}

\author{
Mehdi Bavaghar Zaeimi (iD) and Amir Abbas Rassafi \\ Faculty of Engineering, Imam Khomeini International University, Qazvin, Iran \\ Correspondence should be addressed to Mehdi Bavaghar Zaeimi; m_bavaghar@yahoo.com
}

Received 10 March 2021; Revised 26 May 2021; Accepted 17 July 2021; Published 31 July 2021

Academic Editor: Rakesh Mishra

Copyright (c) 2021 Mehdi Bavaghar Zaeimi and Amir Abbas Rassafi. This is an open access article distributed under the Creative Commons Attribution License, which permits unrestricted use, distribution, and reproduction in any medium, provided the original work is properly cited.

\begin{abstract}
Municipal solid waste management (MSW) is a factor that affects environmental pollution and the spread of diseases in cities. Therefore, an efficient MSW management system results in reducing the cost of environmental impact by tackling the processes of waste collection, recycling, and disposal. In this study, a biobjective optimization model is developed which aims to minimize the costs of facility location and transportation planning and the emission of environmental pollutants. Furthermore, to consider the uncertain nature of the problem, demand or the volume of the generated waste is considered as a random parameter. As a result, a stochastic mathematical programming model with probable constraints is developed. To solve and validate the model, the $\mathcal{E}$-constraint approach has been employed. Moreover, for a real-world application of the proposed model, a case study is implemented in Qazvin, Iran. Finally, various problems are solved for different levels of reliability and an efficient MSW system is designed for each of them. Results show that the proposed method was able to achieve Pareto solutions where managers can decide to choose one of them based on their priorities in comparison with the current status. Moreover, results revealed cost and emission would be reduced by increasing confidence level. Finally, a comparison is made between our proposed $\varepsilon$-constraint method and one of the recently used solution approaches.
\end{abstract}

\section{Introduction}

Municipal solid waste (MSW) is the generated solid waste in urban residential areas. MSW includes the generated waste by residential houses, commercial units, industrial sectors, and institutional units such as schools, hospitals, care centers, and public centers such as streets, markets, bus stops, and parks $[1,2]$.

In this study, an integrated location-allocation problem for the municipal waste collection vehicles is studied [3]. For each level, including collection, recycling, and disposal, a set of facilities is selected and transportation planning is set among the different levels.

This study aims to develop an efficient planning system for the collection and transportation of municipal waste $[4,5]$. For this purpose, vehicle routing is one of the main components of the proposed mathematical model while considering the uncertain nature of the demand in the problem. As a result, the outcomes of the research can be implemented in municipal organizations with the least degree of deviation.

Routing the waste collection vehicles and their allocation and considering the various types of waste are the main challenges that municipalities usually face in planning for municipal waste collection. Using these concepts and analyzing the results, various policies can be easily evaluated and the optimal policy can be selected $[6,7]$.

However, a high degree of uncertainty in the amount of generated waste in municipal areas is likely to result in the infeasibility and inefficiency of the problem in static and deterministic conditions [8]. Accordingly, in the next step, the problem is analyzed employing stochastic programming. 
All in all, the purpose of this study is to do the following:

(i) Increase the efficiency of the waste collection system

(ii) Minimize the location and construction costs of required facilities for municipal waste processing

(iii) Minimize the required number of vehicles, total transportation costs between facilities, and operating costs of the facility

(iv) Minimize environmental pollution, specifically greenhouse gas emissions which are generated by various facilities and transportation systems

In the following, the relevant literature of the research is reviewed. The deterministic problem and proposed modeling are presented in Section 3. Stochastic modeling of the problem using the probabilistic programming approach is presented in Section 4. Section 5 includes validation of the proposed model and computational results of the research based on the case study, and finally, conclusions and future suggestions are presented in Section 6.

\section{Literature Review}

In this section, the relevant literature on location problems and vehicle routing problems for municipal waste collection is reviewed.

Huang and Lin [9] proposed a vehicle planning for the municipal waste collection system with a specific "Removing waste from the ground"policy. In this policy, people are responsible for transporting household wastes into the collection vehicles. The purpose of their study was to cover all demand points and to employ an innovative method to optimize routes and the number of vehicles. Kinobe et al. [10] presented a novel and compound approach to optimize waste collection and disposal in the city of Kampala. Their methodology includes the use of Geographic Information System (GIS) tools to optimize total traveled distance, number of trips, and the total spent time on waste collection. This resulted in maximizing the total volume of collected waste, large savings, and environmental conservation. Finally, they were able to determine the appropriate fleet size with the required capacity, taking into account the reduction of fuel consumption and emissions from vehicles to collect waste using GIS tools. There are some other studies that analyzed different aspects of waste management scenarios, such as Suja et al. [11] and Fei-Baffoe et al. [12], who investigated e-waste management scenarios in Malaysia and MSW in Ghana, respectively.

Inghels et al. [13] presented a multipurpose transportation model for designing a municipal solid waste transportation service network. In their research, they studied the possibility of using multipurpose trucks and inland water transport instead of using transport trucks to send bulk household waste from collection centers to waste processing facilities. The proposed model is a dynamic planning model at the tactical level that aims to minimize total transportation, environmental and social costs. By implementing their model in a case study, they proved that the proposed transportation system leads to cost savings.
Akhtar et al. [14] developed a backtracking search algorithm (BSA) to solve VRP models of municipal waste collection problems. The obtained results indicated the suitable performance of the proposed algorithm. Dotoli and Epicoco [15] studied the vehicle routing problem for collecting hazardous municipal waste. They considered traveled distance constraints, time windows, and availability of vehicles in their model and solved a case study to evaluate the applicability of their model.

Jun Zhao et al. [16] proposed a multiobjective continuous network flow model to locate various waste facilities for regional hazardous waste and find the transportation route among them. Their goal was to minimize the total cost and risk. They evaluated their model in a hypothetical case and a realistic one in the Sichuan province of China. López-Sánchez et al. [17] developed a multiobjective optimization algorithm based on the variable neighborhood descent (VND) method to solve the waste collection problem in a city in southern Spain. The goals were to minimize total travel costs and to balance the vehicle routes. They solved the problem and presented the obtained computational results as Pareto front. Hannan et al. [18] developed a VRP model for waste collection by constructing the optimal routes. To solve their problem, they developed a metaheuristic algorithm to solve the examples of the literature. The special feature of this problem was the consideration of weekly planning. Tirkolaee et al. [6] developed a solid arc routing problem by considering the work shift of drivers and crew. To solve the proposed problem and validate their mathematical model, they designed random examples and solved them using the exact method and simulated annealing algorithm. Nowakowski et al. [19] studied a VRPTW problem for collecting mobile e-waste based on the demand records. In their proposed methodology, they considered the possibility of using a comprehensive online communication system for people to register the demand for electronic waste collection and data recording. They solved the VRPTW problem by employing metaheuristic algorithms. Their goal was to determine the required number of collection vehicles, plan the routing of vehicles, collect household waste on time, and minimize collection costs.

Pablo A. Miranda et al. [20] formulated a waste collection problem for a set of nearby islands as a MIP model considering two strategies that were designed for a year. They evaluated the proposed model in a case study in the south of Chile. Rabbani et al. [21] developed a nondominated sorting genetic algorithm II (NSGA-II) and multiobjective PSO (MOPSO) to solve the multiobjective location-routing problem (LRP) of a hazardous waste considering all types of incompatible wastes. The objectives of their proposed model included minimizing the total cost, minimizing the transportation risks of hazardous waste, and minimizing the risk of landfills and waste processing in residential areas. Goli et al. [22] developed a hybrid artificial intelligence and metaheuristic algorithms for dairy products' demand prediction. Particle Swarm Optimization (PSO), Genetic Algorithm (GA), Invasive Weed Optimization (IWO), and Cultural Algorithm (CA) are applied as well as 
Adaptive-Neural-based Fuzzy Inference System (ANFIS), and Support Vector Regression (SVR).

A triobjective MILP model was offered by Olapiriyakul et al. [23] to design a sustainable MSW network. They considered sustainability issues such as land use and public health impacts and investigated a real case study in Thailand. Asefi et al. [24] proposed a MILP formulation to solve the HFVRP problem considering the variable size of the fleet for integrated MSW collection. Their goal was to minimize the transportation costs and total deviation from the fair distribution of goods in the transportation stations. Finally, they employed lexicographic and ideal programming methods to solve the proposed model. Habib et al. [25] designed a large-scale natural disaster waste management system considering a sustainability index. They proposed a fuzzy multiobjective planning model to model the supply chain for the processing of waste generated after the disaster. Wei et al. [26] developed a hybrid algorithm based on an artificial bee colony algorithm to solve the waste collection problem in the form of a CVRP problem considering intermediary disposal. The achievement of their research was a 7.16\% reduction in carbon emissions compared to the traditional collection model. More studies performed on the application of routing problems for MSW collection application can be found in Babaee Tirkolaee et al. [27]; Babaee Tirkolaee et al. [28]; Babaee Tirkolaee et al. [29] Aliahmadi et al. [30]. Goli et al. [31] applied a robust optimization approach for a multiobjective product portfolio problem. They developed the multiobjective invasive weed optimization (MOIWO) algorithm to solve the proposed model. Goli and Malmir [32] developed an allocationrouting model for vehicles in a disaster area under uncertainty. They proposed a GRASP algorithm and a harmony search algorithm to find solutions. Goli et al. [33] developed an integrated approach to predict demand for dairy products. They developed grey wolf optimization (GWO), invasive weed optimization (IWO), cultural algorithm (CA), and particle swarm optimization (PSO).

Recently, Tirkolaee et al. [34] presented a study to develop a robust optimization model to design a municipal waste management system taking into account economic and environmental indicators under uncertainty. They evaluated and validated the proposed model employing a robust optimization approach and conducting a case study. In another study, Tirkolaee et al. [8] developed a sustainable routing location model for managing the collection and timely disposal of COVID-19-related hospital waste during the pandemic. They also employed goal programming and a chance-constraints fuzzy approach to deal with the multiobjectiveness and uncertainty of the problem. Pourhejazy et al. [35] developed a two-index CGRP formulation model considering traditional (door-to-door) and on-call collection methods for electrical and electronic equipment wastes. They aimed to maximize the profit with acceptable service quality. Goli et al. [36] developed a fuzzy programming model for the integrated cell formation and production scheduling problem. They proposed a hybrid genetic algorithm and whale optimization algorithm to solve the problem considering automated guided vehicles and human factors. Pahlevan et al. [37] developed a multiobjective sustainable closed-loop supply chain using products' life cycle in the aluminum industry. Some metaheuristic algorithms including multiobjective grey wolf optimizer (MOGWO), the multiobjective red deer algorithm (MORDA), and augmented epsilon constraint (AEC) are used to obtain Pareto solutions.

On the other hand, there are some existing transportation planning models such as 'MyRouteOnline' software [38] and Route View ProTM software [39] which exclusively deal with routing decisions. Moreover, other types of routing decisions in the field of MSW management can be found in the literature addressing the application of Geographic Positioning System (GPS) in vehicles. In this regard, you may see Faccio et al. [40]. In this study, there is no routing decision practically, but the transportation planning is conducted along with locational and allocation decisions. In other words, in transportation planning models, the scheduling of vehicles and service sequence not considered. The only important point is the determination of appropriate vehicles and the number of products to be transported between two facilities. In fact, we tried to address the strategic and operational decisions in our proposed MSW system.

Table 1 summarizes the current research and the most important studies in the literature.

\section{Problem Definition}

In this section, the proposed biobjective mathematical model for facility location and transportation planning of municipal waste management network is developed. The intended network consists of five levels which include different municipal waste generation centers, waste collection centers, recycling facilities, disposal centers, and shopping centers of recycled material. The model aims to minimize the total cost and minimize gas emissions and environmental pollutions. Moreover, the location of waste generation centers and shopping centers of recycled waste is fixed, and the goal is to locate collection, recycling, and disposal facilities due to capacity constraints.

The proposed network is considered a dynamic (multiperiod) supply chain. To better understand the research problem, Figure 1 illustrates the network for the collection, transportation, disposal, and recycling of municipal solid waste. The main assumptions of the problem are as follows:

(i) The model is multiperiod and decisions are made in a time horizon.

(ii) The model contains five levels such as waste generators, collection centers, recycling centers, disposal centers, and shopping centers of recycled waste. But, the locations of the three levels are just determined by the model consisting of collection, disposal, and recycling centers.

(iii) The capacities are limited.

(iv) There is a setup fee for the facilities. 


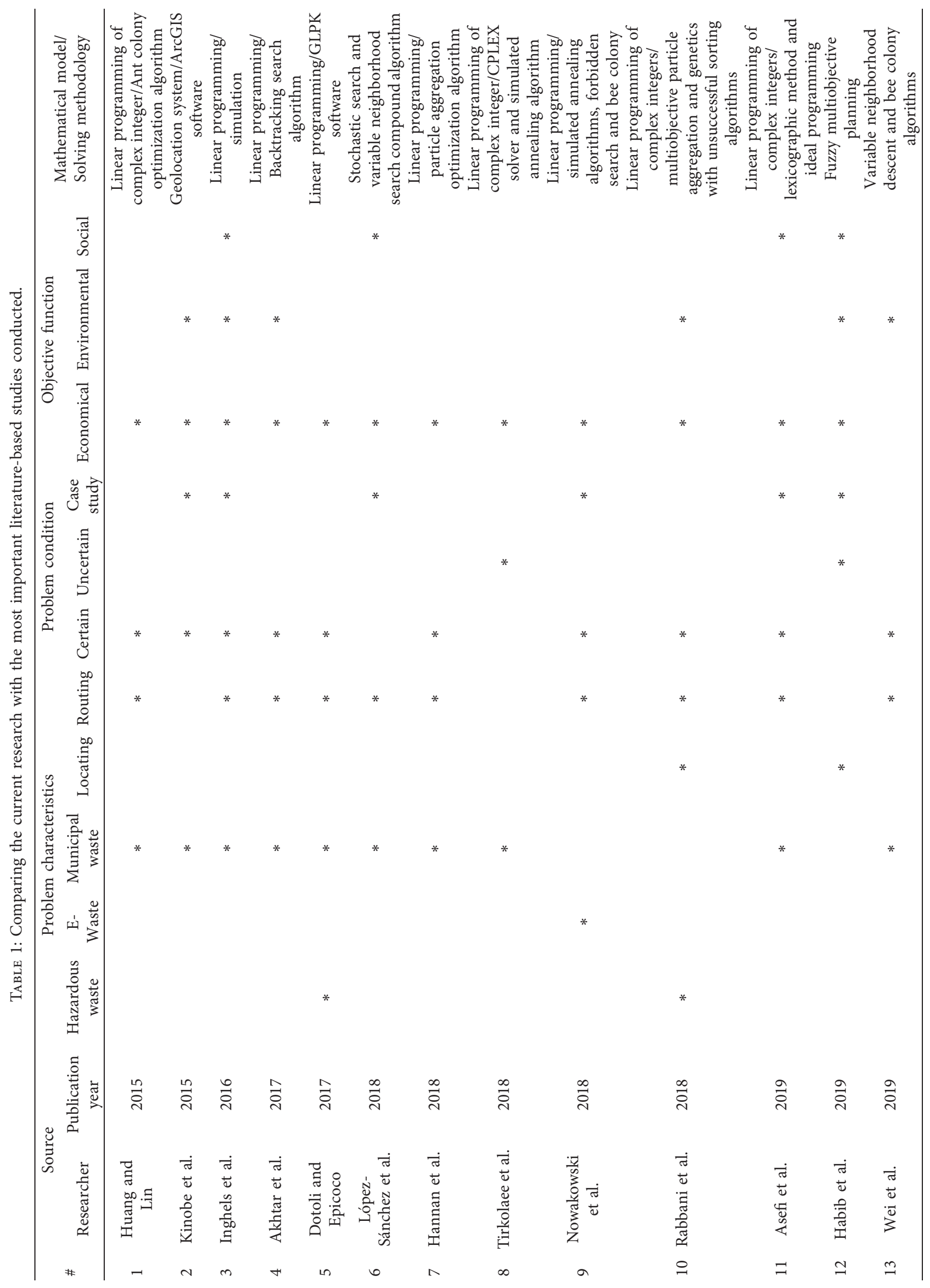




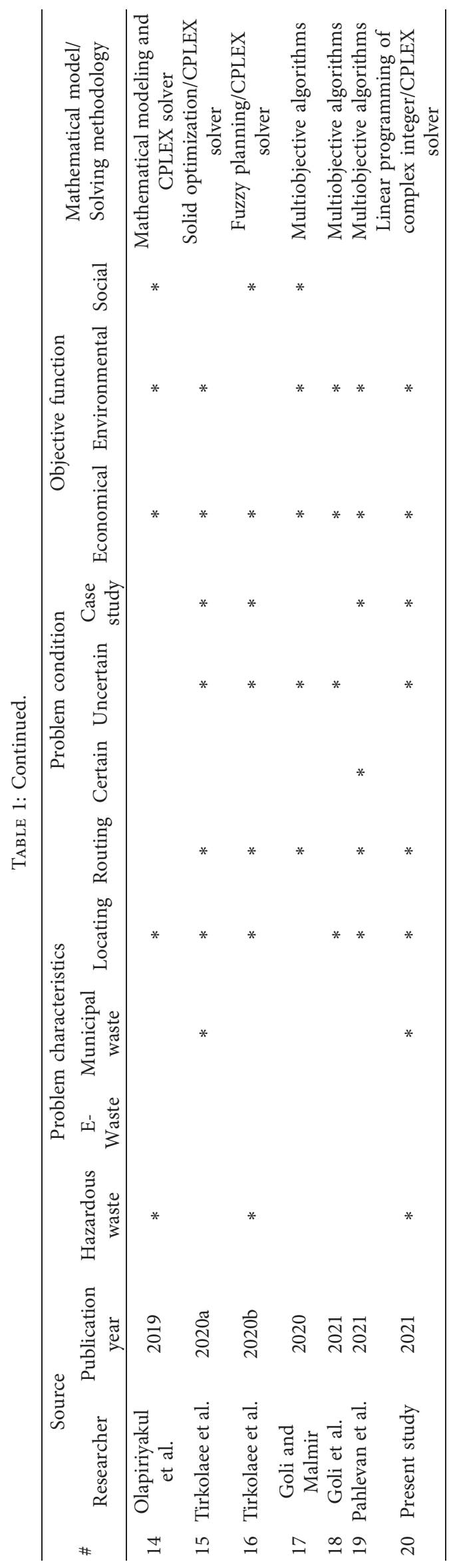




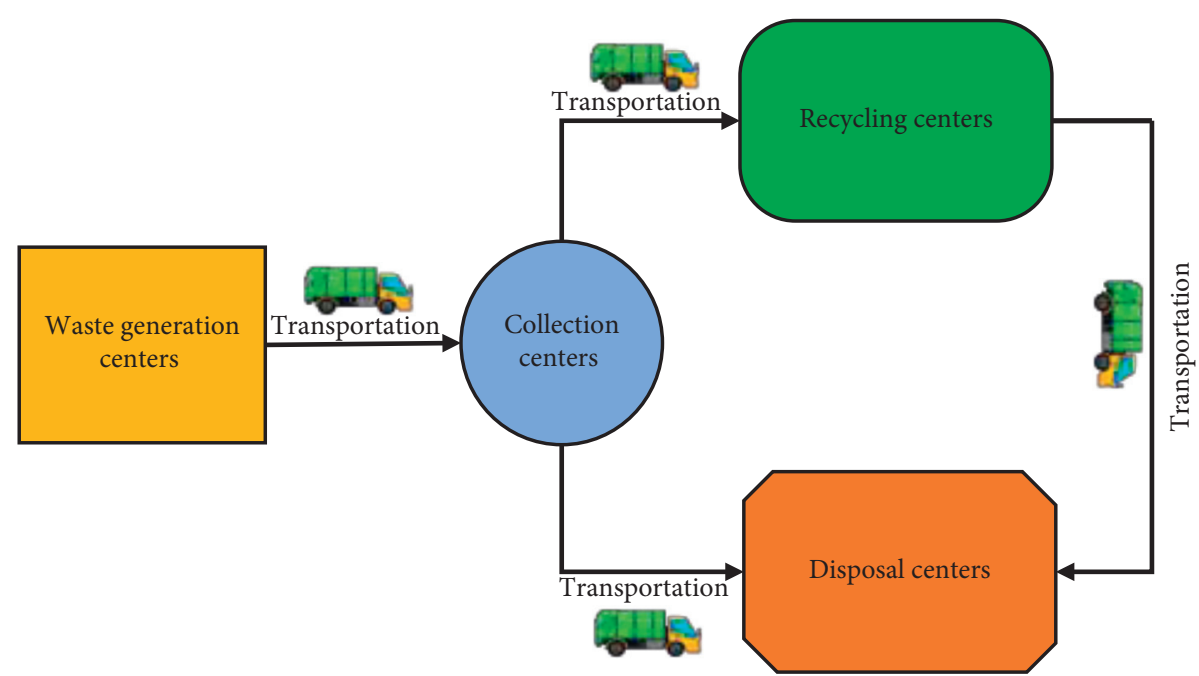

FIgUre 1: Proposed network for an efficient MSW system.

(v) The amount of greenhouse gas emissions is proportional to the operational activities of the facilities and transportation between different levels.

(vi) The amount of produced gas is a function of distance and the amount of transported product.

(vii) The demand of each waste generating center is met by a collection center during the service period.

(viii) In each period, each facility is only assigned to one related facility.

3.1. Mathematical Model. Initially, the following notation is used to formulate the proposed mathematical model:

Indices and sets

M: Waste generation centers;

$W$ : Collecting centers;

$D$ : Recycling centers;

C: Disposal centers;

$K$ : shopping centers of recycled waste;

$T$ : Planning periods;

$m$ : Index of waste generation centers $(m=1,2, \ldots, M)$;

$w$ : Index of collecting centers $(w=1,2, \ldots, W)$;

$d$ : Index of recycling centers $(d=1,2, \ldots, D)$;

$c$ : Index of disposal centers $(c=1,2, \ldots, C)$;

$k$ : Index of shopping centers of recycled wastes $(k=1$,

$2, \ldots, K)$;

$t$ : Index of periods $(t=1,2, \ldots, T)$;

$h$ : Index of transportation system $(h=1,2, \ldots, H)$.

Parameters

$F c_{f}$. Setup fee of facility $f ; f \in\{w, d, c\}$;

$v c_{f t}$ : Variable fees of facility $f$ in period $t ; f \in\{w, d, k, c\}$;

$C_{m w t h}$ : Transporting fee of a unit from generating center $m$ to collection center $w$ in period $t$ by transport system $h$;
$C_{w d t h}$ : Transporting fee of a unit from collection center $w$ to recycling center $d$ in period $t$ by transport system $h$;

$C_{w c t h}$ : Transporting fee of a unit from collection center $w$ to disposal center $c$ in period $t$ by transport system $h$;

$C_{d k t h}$ : Transporting fee of a unit from recycling center $d$ to shopping center of recycled waste $k$ in period $t$ by transport system $h$;

dis $s_{f}$ : Distance between facility $f$ and $f$;

$c a_{w t}$ : Capacity of collection center $w$ in period $t$;

$c a_{d t}$ : Capacity of recycling center $d$ in period $t$;

$c a_{c t}$ : Capacity of disposal center $c$ in period $t$;

$c a_{k t}$ : Capacity of shopping center of recycled waste $k$ in period $t$;

$c v_{h}$ : Capacity of transportation system $h$;

dem $_{m t}$ : Demand in waste generation center $m$ in period $t$;

$G_{t}^{f}$ : The amount of emitted gas in the processing of a waste unit in facility $f$ in period $t$;

$G T^{f f^{\prime}}$ : The amount of emitted gas to transport a waste unit from facilities $f$ to $f$ in period $t$ by transport system $h$;

$\alpha_{d t}$ : Percentage of transported waste from recycling center $d$ to disposal centers in period $t$;

M: A large number.

\section{Decision variables}

$X_{w t}$ : If collection center $w$ is set up in period $t$, the value is 1 , otherwise the value is zero.

$X_{d t}$ : If recycling center $d$ is set up in period $t$, the value is 1 , otherwise the value is zero.

$X_{c t}$ : If disposal center $c$ is set up in period $t$, the value is 1. Otherwise, the value is zero.

$x_{m w t h}$ : The amount of transported waste from waste generating center $m$ to collection center $w$ in period $t$ by the transport system $h$ 
$x_{w d t h}:$ The amount of transported waste from waste collection center $w$ to recycling center $d$ in period $t$ by the transport system $h$

$x_{d c t h}$ : The amount of transported waste from recycling center $d$ to disposal center $c$ in period $t$ by the transport system $h$ $x_{d k t h}$ : The amount of transported waste from recycling center $d$ to shopping center of recycled waste $k$ in period $t$ by the transport system $h$

Mathematical model

According to the parameters and variables, the mathematical model of the problem is as follows:

$$
\begin{aligned}
\min Z_{1}= & \sum_{w} \sum_{t} f c_{w} X_{w t}+\sum_{d} \sum_{t} f c_{d} X_{d t}+\sum_{c} \sum_{t} f c_{c} X_{c t}+\sum_{t} \sum_{w} \sum_{m} \sum_{h} v c_{w t} x_{m w t h} \\
& +\sum_{t} \sum_{w} \sum_{d} \sum_{h} v c_{d t} x_{w d t h}+\sum_{t} \sum_{d} \sum_{c} \sum_{h} v c_{c t} x_{d c t h}+\sum_{t} \sum_{d} \sum_{h} \sum_{k} v c_{k t} x_{d k t h} \\
& +\sum_{t} \sum_{w} \sum_{m} \sum_{h}^{h} \operatorname{dis}_{m w} C_{m w t h} x_{m w t h}+\sum_{t} \sum_{w} \sum_{d} \sum_{h} \operatorname{dis}_{w d} C_{w d t h} x_{w d t h}+\sum_{t} \sum_{d} \sum_{c} \sum_{h} \operatorname{dis}_{d c} C_{d c t h} x_{d c t h} \\
& +\sum_{t} \sum_{d} \sum_{h} \sum_{k} \operatorname{dis}_{d k} C_{d k t h} x_{d k t h},
\end{aligned}
$$

$$
\begin{aligned}
& \min Z_{2}=\sum_{t} \sum_{h} \sum_{w} \sum_{m} x_{m w t h} G_{t}^{w}+\sum_{t} \sum_{d} \sum_{w} \sum_{h} x_{w h} G T_{t}^{d} \\
& +\sum_{t} \sum_{c} \sum_{h} \sum_{d} x_{d c t h} G T_{t}^{c}++\sum_{t} \sum_{w} \sum_{m} \sum_{h} \operatorname{dis}_{m w} G T_{t h}^{m w} x_{m w t h}+\sum_{t} \sum_{w} \sum_{d} \sum_{h} \operatorname{dis}_{w d} G T_{t h}^{w d} x_{w d t h} \\
& +\sum_{t} \sum_{d} \sum_{c} \sum_{h} \operatorname{dis}_{d c} G T_{t h}^{d c} x_{d c t h}+\sum_{t} \sum_{d} \sum_{h} \sum_{k} \operatorname{dis}_{d k} G T_{t h}^{d k} x_{d k t h} \\
& \sum_{w} \sum_{h} x_{m w t h}=\operatorname{dem}_{m t}, \quad \forall m, t \\
& \sum_{m} \sum_{h} x_{m w t h} \leq c a_{w t}, \quad \forall w, t \\
& \sum_{w} \sum_{h} x_{w d t h} \leq c a_{d t}, \quad \forall d, t \\
& \sum_{d} \sum_{h} x_{d c t h} \leq c a_{c t}, \quad \forall c, t \\
& \sum_{d} \sum_{h} x_{d k t h} \leq c a_{k t}, \quad \forall t, k, \\
& \sum_{m} \sum_{w} x_{m w t h}+\sum_{w} \sum_{d} x_{w d t h}+\sum_{d} \sum_{c} x_{d c t h}+\sum_{d} \sum_{k} x_{d k t h} \leq c v_{h}, \quad \forall h, t \\
& x_{m w t h} \leq M X_{w t}, \quad \forall m, t, w, h, \\
& x_{w d t h} \leq M X_{d t}, \quad \forall w, d, t, h, \\
& x_{d c t h} \leq M X_{c t}, \quad \forall d, h, t, c, \\
& \sum_{h} \sum_{c} x_{d c t h}=\alpha_{d t} \sum_{w} \sum_{h} x_{w d t h}, \quad \forall d, t \\
& \sum_{h} \sum_{k} x_{d k t h}=\left(1-\alpha_{d t}\right) \sum_{w} \sum_{h} x_{w d t h}, \quad \forall d, t
\end{aligned}
$$




$$
\begin{gathered}
\sum_{m} \sum_{h} x_{m w t h}=\sum_{d} \sum_{h} x_{w d t h}, \quad \forall w, t \\
X_{w t}, X_{d t}, X_{c t} \in\{0,1\}, \quad \forall w, t, h, m, c, k, d . \\
x_{m w t h}, x_{w d t h}, x_{d c t h}, x_{d k t h} \geq 0
\end{gathered}
$$

The objective function (1) is to minimize the costs, including fixed setup fees, variable costs of facilities, and waste transportation costs. The objective function (2) minimizes the gas emission in processing operations of different facilities and the emission of greenhouse gases due to the transportation between different levels. Constraint (3) guarantees that all of the demand must be fulfilled. In other words, the minimum amount of waste sent to collection centers must be equal to demand.

Constraints (4)-(8) indicate the facility's capacity constraints for collection centers, recycling centers, disposal centers, shopping centers of recycled waste, and transportation systems, respectively. Constraints (9)-(11) represent the need to establish collection centers, recycling centers, and disposal centers. Constraint (12) shows the relationship between the amounts of incoming waste from recycling centers to disposal centers in each period. Constraint (13) indicates the relationship between the amounts of incoming waste from recycling centers to the shopping centers of recycled waste in each period. Constraint (14) indicates the relationship between the amount of waste entering the collection centers and the waste exiting from collection centers to disposal centers. Constraint (15) specifies the domain of variables.

3.2. Stochastic Chance-Constraint Programming. To deal with the uncertainty of the parameter, stochastic chanceconstraint programming is investigated. This approach, as a noticeable approach, has been applied by many researchers, which was developed by Charnes and Cooper [41]. Consider $\operatorname{dem}_{m t}$ parameter ( $\sim$ symbol represents the uncertainty). The probability of occurring a constraint is as follows:

$$
P\left(\sum_{w} \sum_{h} x_{m w t h} \geq \tilde{d}_{\mathrm{em}}\right) \geq \alpha_{i}, \quad i=1,2, \ldots, m .
$$

A summary of the chance-constraint programming for maximization and minimization problems is as follows:

$$
\begin{array}{r}
E\left(\sum_{j=1}^{n} c_{k j}^{*} w_{j}-f_{k}^{-}\right)-\varphi^{-1}\left(\alpha_{k}\right) \sqrt{\operatorname{Var}}\left(\sum_{j=1}^{n} c_{k j}^{*} w_{j}-f_{k}^{-}\right) \geq 0, \quad k=1, \ldots, K, \\
E\left(\sum_{j=1}^{n} d_{i j}^{\sim} w_{j}-e_{i}^{\sim}\right)-\varphi^{-1}\left(1-\alpha_{i}\right) \sqrt{\operatorname{Var}}\left(\sum_{j=1}^{n} d_{i j}^{\sim} w_{j}-e_{i}^{\sim}\right) \geq 0, \quad i=1,2, \ldots, m, \\
E\left(\sum_{j=1}^{n} c_{k j}^{*} w_{j}-f_{k}^{+}\right)+\varphi^{-1}\left(\alpha_{k}\right) \sqrt{\operatorname{Var}}\left(\sum_{j=1}^{n} c_{k j}^{*} w_{j}-f_{k}^{+}\right) \leq 0, \quad k=1, \ldots, K .
\end{array}
$$

Based on the constraints of the multiobjective chanceconstraint model at $\alpha \%$ level (confidence level), constraint (3) is changed as follows:

$$
\begin{aligned}
\sum_{w} \sum_{h} x_{m w t h}= & E\left(\operatorname{dem}_{m t}\right) \\
& +\varphi^{-1}\left(1-\alpha_{i}\right) \cdot \sqrt{\operatorname{var}\left(\operatorname{dem}_{m t}\right)}, \quad \forall m, t .
\end{aligned}
$$

\section{Solution Method: $\varepsilon$-Constraint}

In this study, a multiobjective mixed-integer mathematical programming model is proposed. Since the proposed model has two objectives, the solution approach should find the best answer while considering both objectives simultaneously. The $\varepsilon$-constraint method is one of the well-known approaches for dealing with multiobjective problems that solves the problem by transferring all but one of the objective functions to the constraints at each step [42]. The Pareto front can be created employing the $\varepsilon$-constraint method as follows:

$$
\begin{array}{ll}
\operatorname{minimize} & f_{1}(X) \\
& x \in X, \\
\text { subject to } & f_{2}(X) \leq \varepsilon_{2}, \\
& \ldots \\
& f_{n}(X) \leq \varepsilon_{n} .
\end{array}
$$


The steps of the $\varepsilon$-constraint method are as follows:

(i) Select one of the objective functions (cost objective function) as the primary objective function.

(ii) Solve the problem according to each of the objective functions and obtain the optimal values of each objective function separately.

(iii) Divide the interval between two optimal values of the latter objective function (gas emission) into a predetermined number of divisions and obtain a table of values for $\varepsilon_{2}, \ldots, \varepsilon_{\mathrm{n}}$ (iv) Solve the problem with the primary objective function for each of $\varepsilon_{2}, \ldots, \varepsilon_{\mathrm{n}}$ values

(v) Report Pareto solutions.

The $\varepsilon$-constraint approach is implemented in GAMS software. In this method, firstly, each of the objective functions is optimized separately. Thus, the first objective function, i.e., minimization of supply chain costs, is considered as the only objective function of the problem, and according to the existing constraints, the first model (M1) is changed as follows:

$$
\begin{aligned}
\min Z_{1}= & \sum_{w} \sum_{t} f c_{w} X_{w t}+\sum_{d} \sum_{t} f c_{d} X_{d t}+\sum_{c} \sum_{t} f c_{c} X_{c t}+\sum_{t} \sum_{w} \sum_{m} \sum_{h} v c_{w t} x_{m w t h} \\
& +\sum_{t} \sum_{w} \sum_{d} \sum_{h} v c_{d t} x_{w d t h}+\sum_{t} \sum_{d} \sum_{c} \sum_{h} v c_{c t} x_{d c t h}+\sum_{t} \sum_{d} \sum_{h} \sum_{k} v c_{k t} x_{d k t h} \\
& +\sum_{t} \sum_{w} \sum_{m} \sum_{h} \operatorname{dis}_{m w} C_{m w t h} x_{m w t h}+\sum_{t} \sum_{w} \sum_{d} \sum_{h} \operatorname{dis}_{w d} C_{w d t h} x_{w d t h}+\sum_{t} \sum_{d} \sum_{c} \sum_{h} \operatorname{dis}_{d c} C_{d c t h} x_{d c t h} \\
& +\sum_{t} \sum_{d} \sum_{h} \sum_{k} \operatorname{dis}_{d k} C_{d k t h} x_{d k t h}, \\
\sum_{w} \sum_{h} x_{m w t h}= & E\left(\operatorname{dem}_{m t}\right)+\varphi^{-1}\left(1-\alpha_{i}\right) \cdot \sqrt{\operatorname{var}\left(\operatorname{dem}_{m t}\right)}, \quad \forall m, t
\end{aligned}
$$

Equations (4)-(15).

After solving the first model, the best value of the objective function (lowest cost) is stored as the optimal value of the first objective function. Then the second model based on the second objective function, i.e., minimizing the amount of emitted gases, is considered as the only objective of the problem, and according to constraints of the problem, the second model (M2) is created as follows:

$$
\begin{aligned}
\min Z_{2}= & \sum_{t} \sum_{h} \sum_{w} \sum_{m} x_{m w t h} G_{t}^{w}+\sum_{t} \sum_{d} \sum_{w} \sum_{h} x_{w d t h} G T_{t}^{d} \\
& +\sum_{t} \sum_{c} \sum_{h} \sum_{d} x_{d c t h} G T_{t}^{c}++\sum_{t} \sum_{w} \sum_{m} \sum_{h} \operatorname{dis}_{m w} G T_{t h}^{m w} x_{m w t h}+\sum_{t} \sum_{w} \sum_{d} \sum_{h} \operatorname{dis}_{w d} G T_{t h}^{w d} x_{w d t h} \\
& +\sum_{t} \sum_{d} \sum_{c} \sum_{h} \operatorname{dis}_{d c} G T_{t h}^{d c} x_{d c t h}+\sum_{t} \sum_{d} \sum_{h} \sum_{k} \operatorname{dis}_{d k} G T_{t h}^{d k} x_{d k t h} \\
\sum_{w} \sum_{h} x_{m w t h}= & E\left(\operatorname{dem}_{m t}\right)+\varphi^{-1}\left(1-\alpha_{i}\right) \cdot \sqrt{\operatorname{var}\left(\operatorname{dem}_{m t}\right)}, \quad \forall m, t .
\end{aligned}
$$

Equations (4)-(15). 
Finally, the cost objective function is considered as the primary objective function, and the emitted pollutants objective function is considered as a subobjective within the constraints of the problem.

$$
\begin{aligned}
\min Z_{1}= & \sum_{w} \sum_{t} f c_{w} X_{w t}+\sum_{d} \sum_{t} f c_{d} X_{d t}+\sum_{c} \sum_{t} f c_{c} X_{c t}+\sum_{t} \sum_{w} \sum_{m} \sum_{h} v c_{w t} x_{m w t h} \\
& +\sum_{t} \sum_{w} \sum_{d} \sum_{h} v c_{d t} x_{w d t h}+\sum_{t} \sum_{d} \sum_{c} \sum_{h} v c_{c t} x_{d c t h}+\sum_{t} \sum_{d} \sum_{h} \sum_{k} v c_{k t} x_{d k t h} \\
& +\sum_{t} \sum_{w} \sum_{m} \sum_{h} \operatorname{dis}_{m w} C_{m w t h} x_{m w t h}+\sum_{t} \sum_{w} \sum_{d} \sum_{h} \operatorname{dis}_{w d} C_{w d t h} x_{w d t h} \\
& +\sum_{t} \sum_{d} \sum_{c} \sum_{h} \operatorname{dis}_{d c} C_{d c t h} x_{d c t h}+\sum_{t} \sum_{d} \sum_{h} \sum_{k} \operatorname{dis}_{d k} C_{d k t h} x_{d k t h} \\
\sum_{w} \sum_{h} x_{m w t h}= & E\left(\operatorname{dem}_{m t}\right)+\varphi^{-1}\left(1-\alpha_{i}\right) \cdot \sqrt{\operatorname{var}\left(\operatorname{dem}_{m t}\right)}, \quad \forall m, t . \\
\text { Equations }(4)-(15), &
\end{aligned}
$$

\section{Case Study}

In this section, the proposed solution method is validated by implementing a case study in one district of Qazvin, Iran. For this purpose, a densely populated area is considered, which is shown in Figure 2. The required data is estimated using the consultation of an expert team from the local office of Qazvin Municipality. Using these data, two sample problems are generated to fully investigate the proposed mathematical model. Then, different levels of confidence are considered in the problem. The size of problems and values of the parameters are presented in Tables 2 and 3.

\section{Computational Results}

In this section, the case study is solved based on the proposed model. To solve the proposed model, the CPLEX solver of GAMS software is used as an efficient tool in optimal problem-solving. Regarding the proposed solution approach, firstly, the optimal values of two objective functions are obtained in different confidence levels and reported in Tables 4 and 5 .

According to Tables 4 and 5, the optimal value of each objective function has been obtained for different confidence levels for two sample problems. Then, according to the proposed solution approach, the interval between the minimum and maximum amounts of emitted pollutants is divided into a predetermined number of points (divisions). In this research, the number of points (divisions) is equal to 6. Tables 6 and 7 show the $\varepsilon$ values for each breakpoint for the two sample problems, respectively.

Finally, the main problem is solved for each breakpoint. The best possible value of the first objective function for different values of emitted pollutants for the two problems is presented in Tables 8 and 9, respectively.

The obtained Pareto solutions of the first sample problem in three confidence levels are shown in Figure 3.
The obtained Pareto solutions of the second sample problem in three confidence levels are shown in Figure 4.

In order to evaluate the obtained Pareto solutions and to select the best solution, the reference point approach (RPA) has been used. Deb and Sunder [43] proposed this approach to determine the best point in the multiobjective problems. The main idea of this method is to identify the solutions which are close to the reference point. Normal Euclidean distance $\left(\operatorname{dev}_{p}\right)$ between two nondominated solutions is obtained by the following equation:

$$
\operatorname{dev}_{p}=\sqrt{\sum_{o=1}^{O}\left(\frac{f_{o}-z_{o}}{f_{o}^{\max }-f_{o}^{\min }}\right)^{2}}, \quad \forall p=1,2, \ldots, 6,
$$

In equation (24), $f_{o}$ represents the value of objective function $o, z_{o}$ represents the value of the reference point, and $f_{o}^{\max }$ and $f_{o}^{\min }$ represent the maximum and minimum values of objective function $o$, respectively. The value of the reference point is determined based on the importance of the objective function in Table 10 .

As shown in Table 10, the two objective functions are of equal importance. Accordingly, the value of $d e v_{p}$ for each sample problem is obtained and reported in Tables 11 and 12.

According to Tables 11 and 12, the best Pareto solution is obtained regarding the least value of $\operatorname{dev}_{p}$. Table 13 shows the best Pareto solutions for the two sample problems. The runtime values for sample problems 1 and 2 are 5.27 and 79.05 seconds, respectively.

Finally, a sensitivity analysis is performed on the values of the objective function for different confidence levels based on the results in Table 13. This analysis is highly important as it directly affects supply chain costs and emitted pollutants. Depending on the confidence level, the decision-maker can obtain the number of costs and emitted pollutants and choose the desired confidence level according to their 


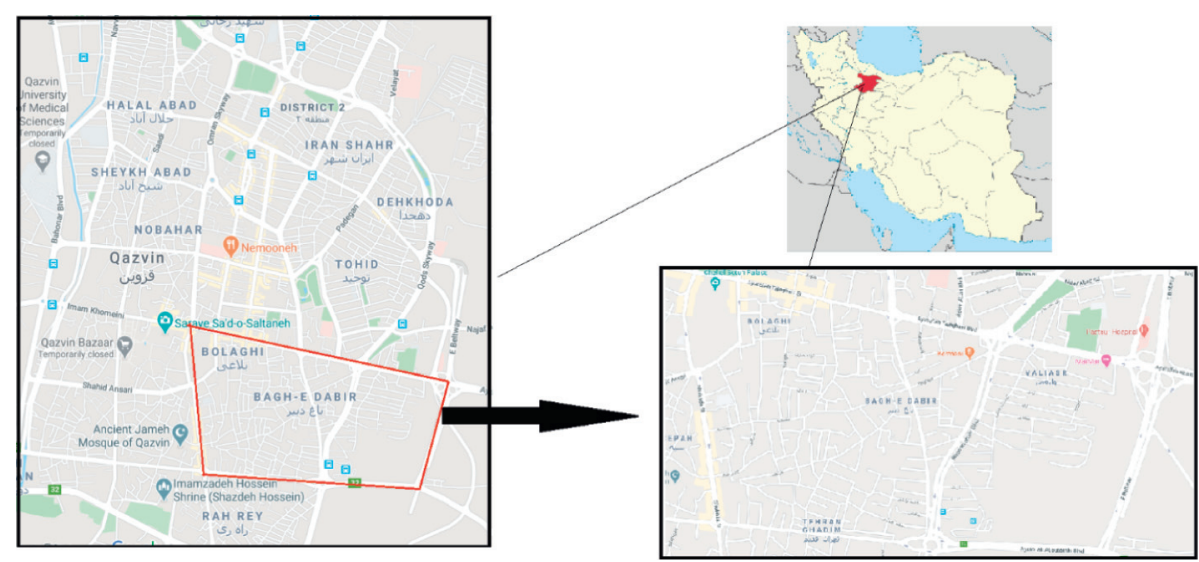

Figure 2: Case study area.

TABle 2: The features of the case study problems.

\begin{tabular}{lccccccc}
\hline Problem no & $M$ & $W$ & $D$ & $C$ & $K$ & $T$ & \\
\hline 1 & 3 & 5 & 8 & 10 & 2 & 5 \\
2 & 6 & 7 & 12 & 12 & 5 & 10 & 7 \\
\hline
\end{tabular}

TABLE 3: Values of input parameters of the problem.

\begin{tabular}{|c|c|c|c|}
\hline Parameters & Values & Parameters & Values \\
\hline$d e m_{m t}$ & Uniform $(15,60)$ & $G_{t}^{f}$ & Uniform $(0.001,0.002)$ \\
\hline$F c_{c}$ & Uniform $(4000,10000)$ & $G T^{f^{t}}{ }_{t h}$ & Uniform $(0.001,0.002)$ \\
\hline$F c_{w}$ & Uniform $(4000,10000)$ & $\alpha_{d t}$ & Uniform $(0.3,0.6)$ \\
\hline$F c_{d}$ & Uniform $(4000,10000)$ & $d i s_{f f}$ & Uniform $(4,4.9)$ \\
\hline$v c_{m t}$ & Uniform $(128,370)$ & $c a_{w t}$ & Uniform $(90,1100)$ \\
\hline$v c_{w t}$ & Uniform $(105,380)$ & $c a_{d t}$ & Uniform $(80,1200)$ \\
\hline$v c_{d t}$ & Uniform $(55,370)$ & $c a_{c t}$ & Uniform $(110,900)$ \\
\hline$v c_{c t}$ & Uniform $(82,395)$ & $c a_{k t}$ & Uniform $(100,1000)$ \\
\hline$C_{m w t h}$ & Uniform $(10,30)$ & $C_{w c t h}$ & Uniform $(1,10)$ \\
\hline$C_{w d t h}$ & Uniform $(10,30)$ & $C_{d k t h}$ & Uniform $(1,10)$ \\
\hline
\end{tabular}

Table 4: Output of the first sample problem.

\begin{tabular}{lcccc}
\hline Confidence level & \multicolumn{2}{c}{ Min $Z_{1}$} & & Min $Z_{2}$ \\
& Cost & Pollution level & Cost & 9.67 \\
\hline 0.6 & 371670.2 & 13.68 & 1444903.4 & 9.59 \\
0.8 & 369313.6 & 13.57 & 1440699 & 9.5 \\
1 & 367157.4 & 13.46 & 1436494.7 & \\
\hline
\end{tabular}

TABLE 5: Output of the second sample problem.

\begin{tabular}{lcccc}
\hline Confidence level & \multicolumn{2}{c}{ Min $Z_{1}$} & & Min $Z_{2}$ \\
& Cost & Pollution level & Cost & Pollution level \\
\hline 0.6 & 1018066.1 & 51.9 & 3313846.7 & 34.68 \\
0.8 & 1010360.2 & 51.52 & 3300598.5 & 34.42 \\
1 & 1002547.6 & 51.16 & 3287350.2 & 34.15 \\
\hline
\end{tabular}

TABLE 6: Breakpoint values for the first sample problem.

\begin{tabular}{lcccccc}
\hline \multirow{2}{*}{ Confidence level } & \multicolumn{5}{c}{ Breakpoints } \\
& 1 & 2 & 3 & 4 & 5 \\
\hline 0.6 & 9.67 & 10.47 & 11.27 & 12.07 & 12.87 & 13.68 \\
0.8 & 9.59 & 10.39 & 11.19 & 11.99 & 12.79 & 13.57 \\
1 & 9.5 & 10.29 & 11.08 & 11.87 & 12.66 & 13.46 \\
\hline
\end{tabular}


TABLE 7: Breakpoint values for the second sample problem.

\begin{tabular}{lcccccc}
\hline \multirow{2}{*}{ Confidence level } & \multicolumn{5}{c}{ Breakpoints } \\
& 1 & 2 & 3 & 4 & 5 \\
\hline 0.6 & 34.68 & 38.12 & 41.56 & 45 & 48.44 & 51.9 \\
0.8 & 34.42 & 37.84 & 41.26 & 44.68 & 51.1 & 52 \\
1 & 34.15 & 37.55 & 40.95 & 44.35 & 47.75 & 51.16 \\
\hline
\end{tabular}

TABLE 8: The value of the cost objective function for breakpoints of the first sample problem.

\begin{tabular}{lcccccc}
\hline \multirow{2}{*}{ Breakpoint } & \multicolumn{2}{c}{0.6} & \multicolumn{2}{c}{0.8} & $Z_{1}$ & \multicolumn{1}{c}{$Z_{1}$} \\
\hline 1 & $Z_{1}$ & $\varepsilon$ & 9.67 & 709419.5 & 9.59 & 699449.4 \\
2 & 725053.6 & 10.47 & 421943.8 & 10.39 & 419853.7 \\
3 & 424888.7 & 11.27 & 391979.3 & 11.19 & 390056.8 & 10.29 \\
4 & 394819.9 & 12.07 & 376640.5 & 11.99 & 11.08 \\
5 & 379373.7 & 12.87 & 371419.9 & 12.79 & 374772.4 \\
6 & 373773.1 & 13.68 & 369339.2 & 13.56 & 369242.2 \\
\hline
\end{tabular}

TABLE 9: The value of the cost objective function for breakpoints of the second sample problem.

\begin{tabular}{|c|c|c|c|c|c|c|}
\hline \multirow{2}{*}{ Breakpoint } & \multicolumn{2}{|c|}{0.6} & \multicolumn{2}{|c|}{0.8} & \multicolumn{2}{|c|}{1} \\
\hline & $Z_{1}$ & $\varepsilon$ & $Z_{1}$ & $\varepsilon$ & $Z_{1}$ & $\varepsilon$ \\
\hline 1 & 1992182.6 & 34.69 & 1952139.7 & 34.42 & 1953162.8 & 34.15 \\
\hline 2 & 1174490.2 & 38.12 & 1164976.9 & 37.84 & 1155993 & 37.55 \\
\hline 3 & 1067692.5 & 41.56 & 1059660 & 41.26 & 1051473.3 & 40.95 \\
\hline 4 & 1029027 & 45 & 1021402.1 & 44.68 & 1013800.5 & 44.35 \\
\hline 5 & 1019686.4 & 48.44 & 1010148.4 & 48.1 & 1004430.3 & 47.75 \\
\hline 6 & 1018066.3 & 51.9 & 1009410.2 & 51.52 & 1002548 & 51.16 \\
\hline
\end{tabular}

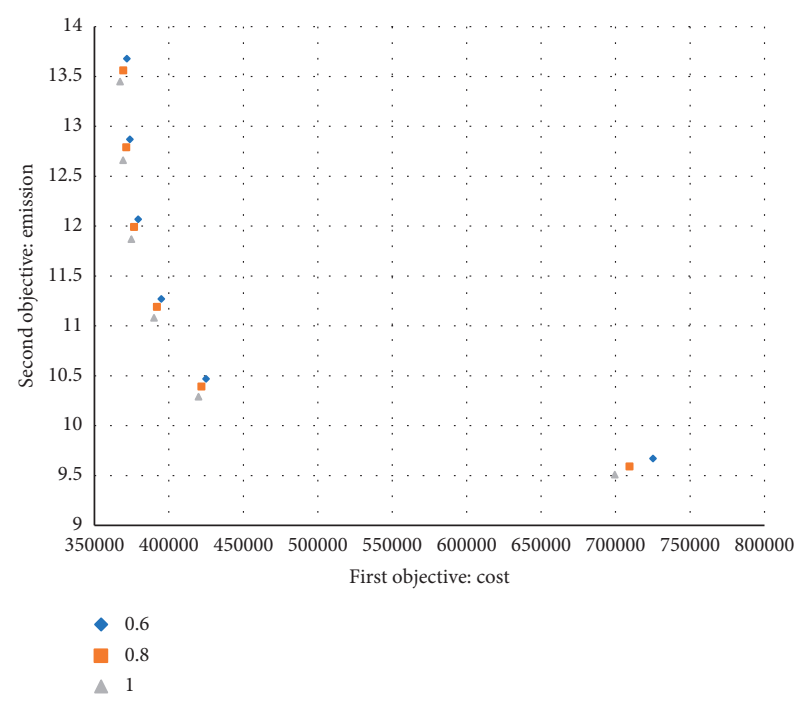

Figure 3: Pareto solution of the first sample problem for $\alpha=0.6$, 0.8 , and 1 .

priority. As shown in Figure 5, by increasing the confidence level, the cost of the whole chain decreases.

Furthermore, Figure 6 shows the changes of the emitted pollutants objective function for the various confidence levels for the two sample problems. It is observed that increasing the confidence level leads to the reduction of pollution.

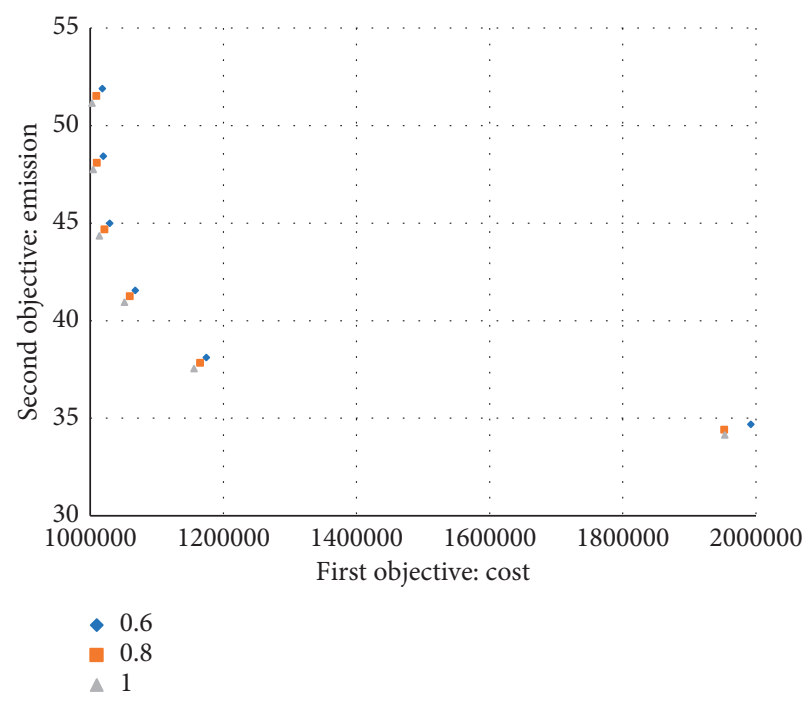

FIGURE 4: Pareto solution of the second sample problem for $\alpha=0.6$, 0.8 , and 1 .

6.1. Comparison with the Current Status. In order to further assess the efficiency and applicability of our proposed methodology, we asked the experts of the given district in Qazvin Municipality to estimate the values of the objective functions in the first sample problem. Moreover, six days of a week starting from April 3, 2021, are regarded as the planning horizon. The confidence level of 1 is also 
TABLE 10: Reference point values.

\begin{tabular}{lr}
\hline Objective function & Reference point \\
\hline$z_{1}$ & $f_{1}^{\min / 1.05}$ \\
$z_{2}$ & $f_{2}^{\min / 1.05}$ \\
\hline
\end{tabular}

TABLE 11: The value of $\operatorname{dev}_{p}$ for each breakpoint for the first sample problem.

\begin{tabular}{lccr}
\hline Breakpoint & 0.6 & 0.8 & 1 \\
\hline 1 & 1.06 & 1.06 & 1.06 \\
2 & 0.37 & 0.38 & 0.38 \\
3 & 0.53 & 0.53 & 0.53 \\
4 & 0.72 & 0.72 & 0.72 \\
5 & 0.91 & 0.92 & 0.92 \\
6 & 1.11 & 1.12 & 1.12 \\
\hline
\end{tabular}

TABle 12: The value of $\operatorname{dev}_{p}$ for each breakpoint for the second sample problem.

\begin{tabular}{lccc}
\hline Breakpoint & 0.6 & 0.8 & 1 \\
\hline 1 & 2.24 & 2.25 & 2.24 \\
2 & 1.87 & 1.88 & 1.88 \\
3 & 2.02 & 2.02 & 2.01 \\
4 & 2.19 & 2.2 & 2.19 \\
5 & 2.38 & 2.38 & 2.38 \\
6 & 2.57 & 2.58 & 2.57 \\
\hline
\end{tabular}

Table 13: The best Pareto solutions.

\begin{tabular}{lccccccrrr}
\hline Sample problem & \multicolumn{3}{c}{0.6} & & 0.8 & & \\
$\operatorname{dev}_{p}$ & $Z_{1}$ & $Z_{2}$ & $\operatorname{dev}_{p}$ & $Z_{1}$ & $Z_{2}$ & $\operatorname{dev}_{p}$ & $Z_{1}$ & $Z_{2}$ \\
\hline 1 & 0.37 & 424888.7 & 10.47 & 0.38 & 421943.8 & 10.39 & 0.38 & 419853.7 & 10.29 \\
2 & 1.87 & 1174490.2 & 38.12 & 1.88 & 1164976.9 & 37.84 & 1.88 & 1155993 & 37.55 \\
\hline
\end{tabular}

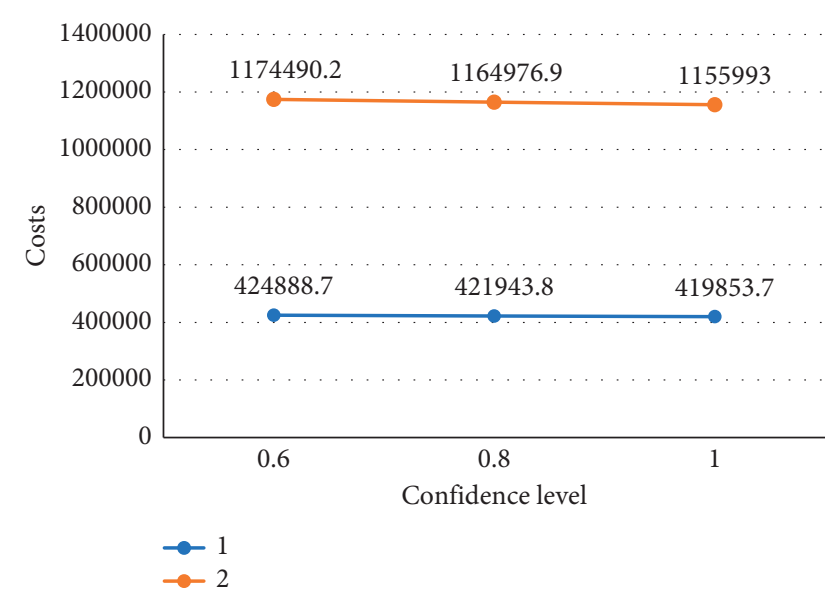

FIGURE 5: The value of the cost objective function for different confidence levels.

considered in order to attain the best reliable results. The comparison of the current and optimal status is represented by Table 14 .

As can be seen in Table 14, our proposed methodology was able to provide a $5.13 \%$ decrease in the total cost and also a $6.20 \%$ decrease in the total pollution emission. It is expected that these savings will be higher in large-scale problems.

6.2. Comparison with WGP Approach. In order to test the efficiency of our solution method, Weighted Goal Programming (WGP) is considered as a rival that was employed by Tirkolaee et al. [34] in a medical waste management system. For more information on the performance of this approach, please see Tirkolaee et al. [34]. The weights of 0.6 and 0.4 are assigned to the $1^{\text {st }}$ and $2^{\text {nd }}$ objective functions based on the experts' opinions. The obtained results from the comparison are shown in Table 15 for both samples.

According to Table 15, it is obvious that different solutions are proposed by $\varepsilon \mathcal{E}$-constraint and WGP methods. The main reason is that the output of WGP is an individual solution, but the $\varepsilon \mathcal{E}$-constraint method generates a Pareto front including Pareto optimal solutions, and finally, the best Pareto solution is obtained by RPA. The important point here is that none of the methods are superior in sample problems. For example, in sample problem 1, WGP obtained a better $Z_{2}$ value while the $\varepsilon \mathcal{E}$-constraint method offers a better $Z_{1}$ value. On the other hand, the average runtime 


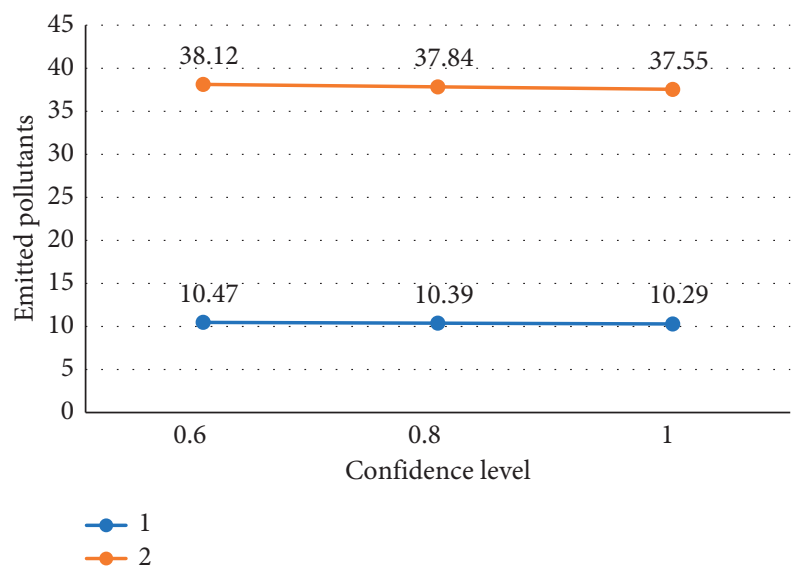

Figure 6: The value of emitted pollutants objective function for different confidence levels.

TABLE 14: Comparison of the current and optimal status.

\begin{tabular}{|c|c|c|c|c|c|}
\hline \multicolumn{2}{|c|}{ Current status } & \multicolumn{2}{|c|}{ Our optimal status } & \multicolumn{2}{|c|}{ Savings percentage } \\
\hline $\begin{array}{l}Z_{1} \\
442546.1\end{array}$ & $\begin{array}{c}Z_{2} \\
10.97\end{array}$ & $\begin{array}{c}Z_{1} \\
419853.7\end{array}$ & $\begin{array}{c}Z_{2} \\
10.29\end{array}$ & $\begin{array}{c}Z_{1} \\
5.13\end{array}$ & $\begin{array}{c}Z_{2} \\
6.20\end{array}$ \\
\hline
\end{tabular}

TABLE 15: Comparison of our proposed $\varepsilon \mathcal{E}$-constraint and WGP method.

\begin{tabular}{|c|c|c|c|c|c|c|}
\hline \multirow{2}{*}{ Sample problem } & \multicolumn{3}{|c|}{ WGP [34] } & \multicolumn{3}{|c|}{$\varepsilon \mathcal{E}$-Constraint } \\
\hline & $Z_{1}$ & $Z_{2}$ & Runtime & $Z_{1}$ & $Z_{2}$ & Runtime \\
\hline 1 & 619678.5 & 9.74 & 2.94 & 419853.7 & 10.29 & 5.27 \\
\hline 2 & 1114023.8 & 38.02 & 46.18 & 1155993 & 37.55 & 79.05 \\
\hline
\end{tabular}

value of WGP is less than the average runtime of the $\varepsilon \mathcal{E}$-constraint method. However, the difference is not tangible and can be ignored. It is very important that the decision-maker applies the more desirable solution method based on its performance.

\section{Conclusions and Future Suggestions}

The management of municipal services is getting more challenging due to the production of solid waste and the occurrence of various social, economic, and environmental incompatibilities related to them. Therefore, it has faced many problems in the collection, transportation, processing, and disposal of such waste.

As mentioned, municipal waste collection is one of the vital issues in the urban area that deals with individual and social health. If waste is not collected in time, it will cause pollution. Therefore, the issue of waste collection planning is of great importance. This research has tried to provide a comprehensive model for the problem of location and use of facilities for waste collection with the aim of minimizing the total costs and pollution caused by the transportation system. Also, in order to evaluate the proposed model and express its feasibility, a case study in Qazvin city, including waste collection planning of an area of the city, has been considered. The obtained results indicate the proper performance of the proposed model so that it can be used for urban waste collection planning.
As a result, in this study, a biobjective mixed-integer planning model was developed considering the real-world assumptions to formulate the integrated location-allocation problem and transportation planning. Due to the uncertainty of demand (amount of generated waste), a stochastic planning approach based on chance constraints was used to deal with the problem of uncertainty. The objectives were to minimize supply chain costs and emitted pollutants by transportation. In order to solve the biobjective model, the $\mathcal{E}$-constraint approach was employed. Afterward, based on the real data, a case study in one district of Qazvin, Iran, was conducted. Finally, the results were analyzed by solving two problems in different sizes and for different confidence levels. Presenting various confidence levels makes decisionmakers obtain the solution with specific cost and emission based on their priority. Results show that increasing confidence level causes to decrease in the total cost and emission amount. Moreover, the comparison with the current status revealed that our proposed methodology can provide significant savings in the total cost and total pollution emission. Finally, a comparison was made between the $\varepsilon \mathcal{E}$-constraint method and WGP approach in order to show the performance of the $\varepsilon \mathcal{E}$-constraint in terms of the $1^{\text {st }}$ and $2^{\text {nd }}$ objective functions, and runtime value.

In this study, a stochastic programming model was used to control uncertainty while other approaches such as robust optimization or fuzzy programming can be applied to compare the results obtained. Furthermore, only beneficial 
and environmental aspects were considered in this research; however, social aspects can be considered by maximizing citizen satisfaction and system reliability [44, 45]. As mentioned, a real case study in small- and medium-size was studied while multiobjective optimization algorithms can be applied to solve highly large-sized problems.

Apart from the optimization recommendations for future research, the application of online tools and Internet-ofThings (IoT) can be studied in the problem to take care of the routing decisions exclusively in the problem. Furthermore, the existing routing software like MyRouteOnline software [38] can be employed to cope with real-time data of routing in an MSW system along with strategic and tactical decisions

\section{Data Availability}

The required data were estimated using the consultation of an expert team from the local office of Qazvin Municipality. The data used to support the findings of this study are included within the article in table format. The complete data source can be accessed for other researchers by author's mail,m_bavaghar@yahoo.com.

\section{Conflicts of Interest}

The authors declare that they have no conflicts of interest.

\section{References}

[1] A. Sharma, R. Ganguly, and A. K. Gupta, "Characterization and energy generation potential of municipal solid waste from nonengineered landfill sites in Himachal Pradesh, India," Journal of Hazardous, Toxic, and Radioactive Waste, vol. 23, no. 4, Article ID 04019008, 2019.

[2] T. D. Nguyen, K. Kawai, and T. Nakakubo, "Drivers and constraints of waste-to-energy incineration for sustainable municipal solid waste management in developing countries," Journal of Material Cycles and Waste Management, vol. 23, pp. 1-10, 2021.

[3] A. N. Srivastava and S. Chakma, "Quantification of landfill gas generation and energy recovery estimation from the municipal solid waste landfill sites of Delhi, India," Energy Sources, Part A: Recovery, Utilization, and Environmental Effects, pp. 1-14, 2020.

[4] V. Yadav and S. Karmakar, "Sustainable collection and transportation of municipal solid waste in urban centers," Sustainable Cities and Society, vol. 53, Article ID 101937, 2020.

[5] A. Taşkın and N. Demir, "Life cycle environmental and energy impact assessment of sustainable urban municipal solid waste collection and transportation strategies," Sustainable Cities and Society, vol. 61, Article ID 102339, 2020.

[6] E. B. Tirkolaee, I. Mahdavi, and M. Mehdi Seyyed Esfahani, "A robust periodic capacitated arc routing problem for urban waste collection considering drivers and crew's working time," Waste Management, vol. 76, pp. 138-146, 2018.

[7] A. Sharma, R. Ganguly, and A. Kumar Gupta, "Impact assessment of leachate pollution potential on groundwater: an indexing method," Journal of Environmental Engineering, vol. 146, no. 3, Article ID 05019007, 2020.

[8] E. B. Tirkolaee, I. Mahdavi, M. M. S. Esfahani, and G. W. Weber, "A robust green location-allocation-inventory problem to design an urban waste management system under uncertainty," Waste Management, vol. 102, pp. 340-350, 2020a.

[9] S. H. Huang and P. C. Lin, "Vehicle routing-scheduling for municipal waste collection system under the "keep trash off the ground" policy," Omega, vol. 55, pp. 24-37, 2015.

[10] J. R. Kinobe, T. Bosona, G. Gebresenbet, C. B. Niwagaba, and B. Vinnerås, "Optimization of waste collection and disposal in Kampala city," Habitat International, vol. 49, pp. 126-137, 2015.

[11] F. Suja, R. Abdul Rahman, A. Yusof, and M. S. Masdar, "Ewaste management scenarios in Malaysia," Journal of Waste Management, vol. 2014, Article ID 609169, 7 pages, 2014.

[12] B. Fei-Baffoe, E. A. Nyankson, and J. Gorkeh-Miah, "Municipal solid waste management in Sekondi-Takoradi metropolis, Ghana," Journal of Waste Management, vol. 2014, Article ID 823752, 9 pages, 2014.

[13] D. Inghels, W. Dullaert, and D. Vigo, "A service network design model for multimodal municipal solid waste transport," European Journal of Operational Research, vol. 254, no. 1, pp. 68-79, 2016.

[14] M. Akhtar, M. A. Hannan, R. A. Begum, H. Basri, and E. Scavino, "Backtracking search algorithm in CVRP models for efficient solid waste collection and route optimization," Waste Management, vol. 61, pp. 117-128, 2017.

[15] M. Dotoli and N. Epicoco, "A vehicle routing technique for hazardous waste collection," IFAC-PapersOnLine, vol. 50, no. 1, pp. 9694-9699, 2017.

[16] J. Zhao, L. Huang, D. H. Lee, and Q. Peng, "Improved approaches to the network design problem in regional hazardous waste management systems," Transportation Research Part E: Logistics and Transportation Review, vol. 88, pp. 52-75, 2016.

[17] A. D. López-Sánchez, A. G. Hernández-Díaz, F. Gortázar, and M. A. Hinojosa, "A multiobjective GRASP-VND algorithm to solve the waste collection problem," International Transactions in Operational Research, vol. 25, no. 2, pp. 545-567, 2018.

[18] M. A. Hannan, M. Akhtar, R. A. Begum, H. Basri, A. Hussain, and E. Scavino, "Capacitated vehicle-routing problem model for scheduled solid waste collection and route optimization using PSO algorithm," Waste Management, vol. 71, pp. 31-41, 2018.

[19] P. Nowakowski, K. Szwarc, and U. Boryczka, "Vehicle route planning in e-waste mobile collection on demand supported by artificial intelligence algorithms," Transportation Research Part D: Transport and Environment, vol. 63, pp. 1-22, 2018.

[20] P. A. Miranda, C. A. Blazquez, R. Vergara, and S. Weitzler, “A novel methodology for designing a household waste collection system for insular zones," Transportation Research Part E: Logistics and Transportation Review, vol. 77, pp. 227-247, 2015.

[21] M. Rabbani, R. Heidari, H. Farrokhi-Asl, and N. Rahimi, "Using metaheuristic algorithms to solve a multiobjective industrial hazardous waste location-routing problem considering incompatible waste types," Journal of Cleaner Production, vol. 170, pp. 227-241, 2018.

[22] A. Goli, H. K. Zare, R. Moghaddam, and A. Sadeghieh, "A Comprehensive Model of Demand Prediction Based on Hybrid Artificial Intelligence and Metaheuristic Algorithms: A Case Study in Dairy Industry," Journal of Industrial and Systems Engineering, vol. 11, pp. 190-203, 2018.

[23] S. Olapiriyakul, W. Pannakkong, W. Kachapanya, and S. Starita, "Multiobjective optimization model for sustainable 
waste management network design," Journal of Advanced Transportation, vol. 2019, Article ID 3612809, 15 pages, 2019.

[24] H. Asefi, S. Shahparvari, P. Chhetri, and S. Lim, "Variable fleet size and mix VRP with fleet heterogeneity in integrated solid waste management," Journal of Cleaner Production, vol. 230, pp. 1376-1395, 2019.

[25] M. S. Habib, B. Sarkar, M. Tayyab, M. W. Saleem et al., "Largescale disaster waste management under uncertain environment," Journal of Cleaner Production, vol. 212, pp. 200-222, 2019.

[26] Q. Wei, Z. Guo, H. C. Lau, and Z. He, “An artificial bee colony-based hybrid approach for waste collection problem with midway disposal pattern," Applied Soft Computing, vol. 76, pp. 629-637, 2019.

[27] E. Babaee Tirkolaee, P. Abbasian, M. Soltani, and S. A. Ghaffarian, "Developing an applied algorithm for multitrip vehicle routing problem with time windows in urban waste collection: a case study," Waste Management \& Research, vol. 37, pp. 4-13, 2019 a.

[28] E. Babaee Tirkolaee, A. Goli, M. Pahlevan, and R. Malekalipour Kordestanizadeh, "A robust bi-objective multi-trip periodic capacitated arc routing problem for urban waste collection using a multiobjective invasive weed optimization," Waste Management \& Research, vol. 37, no. 11, pp. 1089-1101, 2019b.

[29] E. Babaee Tirkolaee, I. Mahdavi, M. M. Seyyed Esfahani, and G. W. Weber, "A hybrid augmented ant colony optimization for the multi-trip capacitated arc routing problem under fuzzy demands for urban solid waste management," Waste Management \& Research, vol. 38, no. 2, pp. 156-172, 2020.

[30] S. Z. Aliahmadi, F. Barzinpour, and M. S. Pishvaee, "A fuzzy optimization approach to the capacitated node-routing problem for municipal solid waste collection with multiple tours: a case study," Waste Management \& Research, vol. 38, no. 3, pp. 279-290, 2020.

[31] A. Goli, H. K. Zare, R. Tavakkoli-Moghaddam, and A. Sadeghieh, "Application of robust optimization for a product portfolio problem using an invasive weed optimization algorithm," Numerical Algebra, Control \& Optimization, vol. 9, no. 2, p. 187, 2019.

[32] A. Goli and B. Malmir, "A covering tour approach for disaster relief locating and routing with fuzzy demand," International Journal of Intelligent Transportation Systems Research, vol. 18, no. 1, pp. 140-152, 2020.

[33] A. Goli, H. Khademi-Zare, R. Tavakkoli-Moghaddam, A. Sadeghieh et al., "An integrated approach based on artificial intelligence and novel meta-heuristic algorithms to predict demand for dairy products: a case study," Network: Computation in Neural Systems, pp. 1-35, 2020.

[34] E. B. Tirkolaee, P. Abbasian, and G. W. Weber, "Sustainable fuzzy multi-trip location-routing problem for medical waste management during the COVID-19 outbreak," The Science of the Total Environment, vol. 756, Article ID 143607, 2021.

[35] P. Pourhejazy, D. Zhang, Q. Zhu, F. Wei, and S. Song, "Integrated E-waste transportation using capacitated general routing problem with time-window," Transportation Research Part E: Logistics and Transportation Review, vol. 145, Article ID 102169, 2021.

[36] A. Goli, E. B. Tirkolaee, and N. S. Aydin, "Fuzzy integrated cell formation and production scheduling considering automated guided vehicles and human factors," IEEE Transactions on Fuzzy Systems, 2021.

[37] S. M. Pahlevan, S. M. S. Hosseini, and A. Goli, "Sustainable supply chain network design using products' life cycle in the aluminum industry," Environmental Science and Pollution Research, pp. 1-25, 2021.

[38] E. Amarachukwu, A. M. Evuti, K. A. Salam, and S. S. Silas, "Determination of waste generation, composition and optimized collection route for university of abuja main campus using "myrouteonline" software," Scientific African, vol. 10, Article ID e00569, 2020.

[39] O. Apaydin and M. T. Gonullu, "Route optimization for solid waste collection: trabzon (Turkey) case study," Global NEST Journal, vol. 9, no. 1, pp. 6-11, 2007.

[40] M. Faccio, A. Persona, and G. Zanin, "Waste collection multi objective model with real time traceability data," Waste Management, vol. 31, no. 12, pp. 2391-2405, 2011.

[41] A. Charnes and W. W. Cooper, "Chance-constrained programming," Management Science, vol. 6, no. 1, pp. 73-79, 1959.

[42] M. Ehrgott and G. Xavier, Multi Objective Combinatorial Optimization Theory, Methodology, and Applications, Multiple Criteria Optimization: State of the Art Annotated Bibliographic Surveys, Springer, Boston, MA, USA, 2003.

[43] K. Deb and J. Sundar, "Reference point based multiobjective optimization using evolutionary algorithms," in Proceedings of the 8th Annual Conference on Genetic and Evolutionary Computation, vol. 12, pp. 635-642, Washington, DC, July 2006.

[44] A. Khakbaz and E. Babaee Tirkolaee, "A sustainable hybrid manufacturing/remanufacturing system with two-way substitution and WEEE directive under different market conditions," Optimization, pp. 1-24, 2021.

[45] N. Jahani, A. Sepehri, H. R. Vandchali, and E. B. Tirkolaee, "Application of industry 4.0 in the procurement processes of supply chains: a systematic literature review," Sustainability, vol. 13 , no. 14 , p. $7520,2021$. 